Review

\title{
High resolution electrophysiological techniques for the study of calcium-activated exocytosis 3
}

\author{
Manfred Lindau \\ 272 Clark Hall, School of Applied and Engineering Physics, Cornell University, Ithaca NY 14853, USA
}

\section{A R T I C L E I N F O}

Article history:

Received 24 October 2011

Received in revised form 9 December 2011

Accepted 10 December 2011

Available online 22 December 2011

\section{Keywords:}

Exocytosis

Transmitter release

Fusion pore

Patch clamp

Capacitance measurement

\begin{abstract}
A B S T R A C T
Background: Neurotransmitters, neuropeptides and hormones are released from secretory vesicles of nerve terminals and neuroendocrine cells by calcium-activated exocytosis. A key step in this process is the formation of a fusion pore between the vesicle membrane and the plasma membrane. Exocytotic fusion leads to an increase in plasma membrane area that can be measured as a proportional increase in plasma membrane capacitance.

Scope of review: High resolution capacitance measurements in single cells, nerve terminals and small membrane patches have become possible with the development of the patch clamp technique. This review discusses the methods of whole cell patch clamp capacitance measurements and their use in conjunction with voltage clamp pulse stimulation and with stimulation by photorelease of caged calcium. It also discusses patch capacitance measurements for the study of single exocytotic events and fusion pore properties in neuroendocrine cells and nerve terminals.

Major conclusions: Capacitance measurements provide high resolution information on the extent and time course of fusion for the characterization of vesicle pools and the kinetics of exocytosis. They allow the characterization of the mode of fusion including distinction of single vesicle full fusion, transient kiss-and-run fusion or multivesicular compound exocytosis. Furthermore, measurement of fusion pore conductances and their dynamic behavior has enabled the characterization of fusion pore properties in a way that resembles the characterization of ion channel function through single channel recordings.

General significance: The combination of patch clamp capacitance measurements with pharmacological and molecular manipulations of exocytosis is emerging as a powerful approach to investigate the molecular mechanisms of calcium-activated exocytotic fusion pore formation. This article is part of a Special Issue entitled Biochemical, biophysical and genetic approaches to intracellular calcium signalling.
\end{abstract}

(c) 2011 Elsevier B.V. All rights reserved.

\section{Introduction}

Many cell types store specific molecules in intracellular vesicles and release the contents of the vesicles in response to an increase of the free intracellular calcium concentration $\left[\mathrm{Ca}^{2+}\right]_{\mathrm{i}}$. The release occurs by exocytosis, the formation of a fusion pore connecting the intravesicular lumen to the extracellular space allowing for release of the vesicular contents [1]. Calcium-activated exocytosis mediates the release of neurotransmitters in synapses, release of neuropeptides and hormones from nerve terminals and neuroendocrine cells as well as exocytotic events in a number of other cell types.

Measurements of exocytosis typically detect the release of molecules from the cell or the change in the cell's plasma membrane area that is the consequence of fusion of secretory vesicles with the

\footnotetext{
it This article is part of a Special Issue entitled Biochemical, biophysical and genetic approaches to intracellular calcium signalling.

E-mail address: ml95@cornell.edu.
}

plasma membrane. Many different detection methods have been used to quantify release or changes in membrane area. In excitable cells, exocytosis in response to $\mathrm{Ca}^{2+}$ entry is rapid and may occur within less than a millisecond [2-4] such that high time resolution techniques are needed.

One method to detect release of neurotransmitters from single vesicles employs recording of postsynaptic currents or postsynaptic potentials utilizing the endogenous receptors of the postsynaptic cell. Indeed, such measurements had provided the first indication of quantal release [5]. Following the ultrastructural observation of "a vesicular or granular component of the synapse" [6] , the vesicular hypothesis of quantal release was put forward [7]. The high time resolution in such measurements comes from the short diffusion distance between the presynaptic release sites and the postsynaptic receptors and from the rapid response of ionotropic receptors, which are ion channels that open with rapid kinetics after binding the respective neurotransmitter molecules. However, the analysis of such measurements is complicated by the fact that multiple mechanisms determine the properties of the measured postsynaptic signals, which are not 
only affected by the presynaptic release but also by the postsynaptic receptors $[8,9]$.

Release of oxidizable transmitters such as dopamine, noradrenaline, or serotonin can be detected electrochemically using the method of amperometry, which directly records the interaction of released molecules with the electrode surface. The first amperometric recordings of single vesicle release events were performed with chromaffin cells using carbon fiber microelectrodes [10]. Single release events manifest themselves as amperometric spikes. These amperometric spikes are often preceded by a 'foot' signal and it was suggested that these foot signals may reflect the slow release of neurotransmitter through a narrow fusion pore [11]. Electrochemical methods to detect release from single vesicles and the analysis of amperometric spikes have been discussed in depth in a number of excellent reviews [12-14] and will therefore not be addressed in much detail here. This review is focused on high resolution techniques to measure calciumactivated exocytosis by patch clamp capacitance measurements. With the focus being on the techniques, it will not be possible to mention the hundreds of excellent studies in which these techniques were applied to address specific questions on the cellular and molecular mechanisms of calcium-activated exocytosis and their regulation.

\section{Capacitance measurements report calcium-activated exocytosis}

The fusion of individual vesicles with the plasma membrane during exocytosis can be measured directly as a change in plasma membrane capacitance. Historically, the first measurements of a capacitance increase associated with exocytosis were reported by Cole who performed measurements with extracellular electrodes on a suspension of egg cells and found that the membrane capacitance was 2.5 times higher in fertilized eggs [15]. At that time, however, the physical basis of this capacitance increase remained unknown until about 20 years later exocytotic cortical granule fusion was observed by electron microscopy [16,17]. The capacitance increase of the egg cell membrane after fertilization was later confirmed by single cell capacitance measurements using microelectrodes [18]. Cortical granule fusion in egg cells is a calcium-dependent exocytotic mechanism and a capacitance increase was reported for Xenopus eggs in response to stimulation with a calcium ionophore [19].

\subsection{Whole cell patch clamp capacitance measurements}

High resolution measurements of calcium-activated exocytosis became possible with the development of the giga-ohm seal patch clamp technique which allows voltage clamp recordings with very low noise from small cells and membrane patches [20]. This was first demonstrated in bovine chromaffin cells in the pioneering work of Neher and Marty [21] who demonstrated that in the whole cell configuration a capacitance increase could be stimulated by depolarizing pulses and that these capacitance changes were linked to calcium currents. It was shown that a chromaffin cell can be represented by the simple equivalent circuit shown in Fig. 1.

During a recording the three parameters of the equivalent circuit may change. The most widely used methods to record changes in membrane capacitance utilize a sine wave voltage and analyze the resulting current. Such a measurement provides in principle two quantities such as amplitude and phase of the current relative to those of the voltage stimulus. In practice, a lock-in amplifier is often used to provide the current amplitudes at two orthogonal phases. When the phase is properly chosen, changes in membrane capacitance appear in only one of the lock-in amplifier outputs $\left(\mathrm{Y}_{\mathrm{C}}\right)$ whereas changes in membrane conductance $\left(G_{M}=1 / R_{M}\right)$ or access resistance appear in the other channel $\left(Y_{R}\right)[21-24]$ :

$\Delta Y_{C}=T^{2} \cdot \omega \Delta C_{M}$

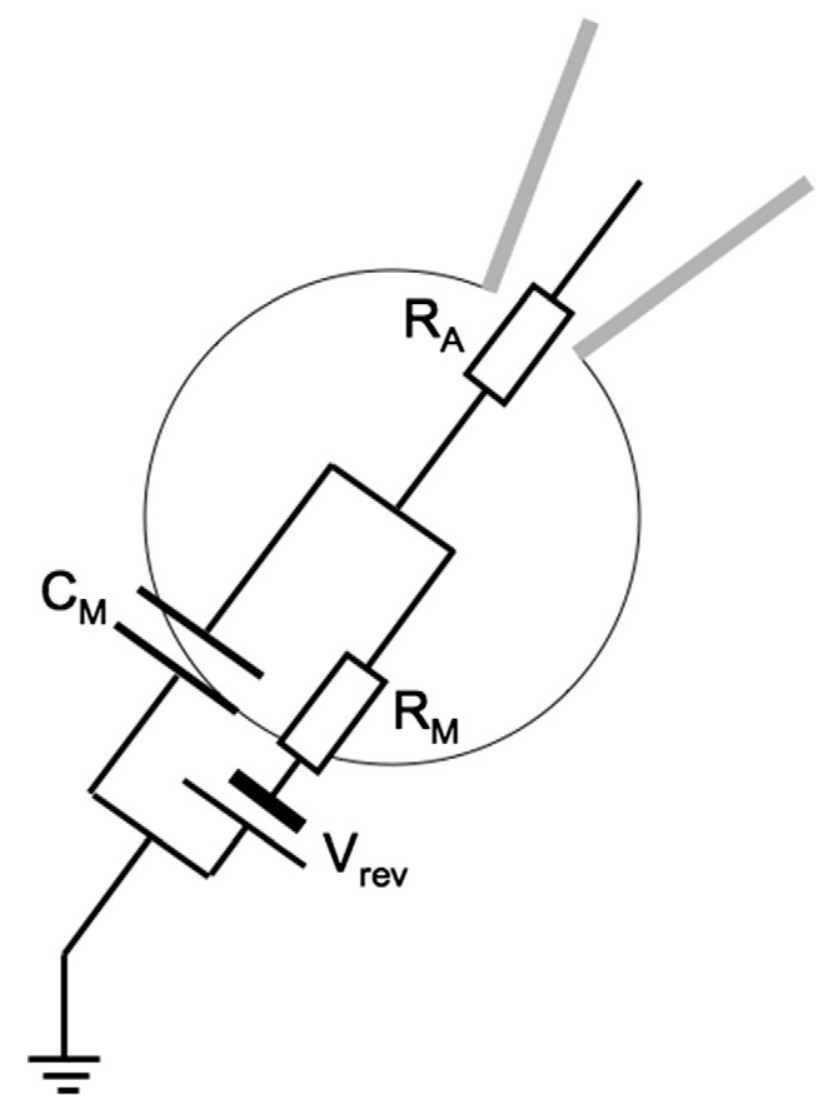

Fig. 1. Whole cell equivalent circuit of a chromaffin cell. The cell membrane is represented by the parallel combination of membrane capacitance $C_{M}$ and membrane resistance $R_{M}$ with the reversal potential $V_{\text {rev }} R_{A}$ is the access resistance of the pipette tip connecting to the pipette electrode to the intracellular space.

$\Delta Y_{R}=T^{2} \cdot\left[\Delta G_{M}+\Delta R_{A}\left(\omega C_{M}\right)^{2}\right]$

Here, $\omega=2 \pi f$ is the angular frequency ( $\mathrm{f}=$ sine wave frequency). $T^{2}$ is a frequency dependent scaling factor that may be determined by changing the capacitance compensation by a small, defined amount. This approach holds as long as all the changes are relatively small and the method has been named "piecewise linear technique".

\subsubsection{The "sine plus dc" method}

When larger changes are being tracked and to provide independent estimates of all three equivalent circuit parameters, a third parameter needs to be measured. In the widely used "sine plus dc" method [22] the holding current $\mathrm{I}_{\mathrm{H}}$ is measured, which provides $R_{m}+R_{A}=\left(V_{H}-V_{\text {rev }}\right) / I_{H}$. Obviously, the chosen holding voltage $V_{H}$ must be sufficiently different from $V_{\text {rev }}$ to obtain a proper signal. Typically, the cell's membrane resistance is very high, in the giga-ohm range, and the precise value of $V_{\text {rev }}$ does not affect the determination of $C_{m}$ very much. In chromaffin cells, a significant decrease of $R_{m}$ typically comes from the opening of $\mathrm{Ca}^{2+}$ - and voltage-dependent potassium channels (BK channels) [25] or from some leak at the pipette-cell seal. Choosing a $\mathrm{Cs}^{+}$rather than $\mathrm{K}^{+}$based intracellular (pipette) solution strongly reduces $\mathrm{K}^{+}$currents and it is usually a good choice to set $\mathrm{V}_{\mathrm{H}}$ to $\sim 70 \mathrm{mV}$ and to assume $\mathrm{V}_{\text {rev }}=0 \mathrm{mV}$. The method is implemented [26] in the Pulse and Patchmaster software (HEKA, Lambrecht, Germany) and is currently the most widely used electrophysiological technique to measure calcium-activated exocytosis.

\subsubsection{Dual frequency methods}

Under conditions where $\mathrm{V}_{\text {rev }}$ changes considerably during the recording and $R_{m}$ is low, or when the equivalent circuit of the cell is 
more complex than that of Fig. 1, methods that use voltage signals composed of more than one frequency may be advantageous. Using a sum of two sine waves with different frequency provides 4 measured quantities and the three parameters of the equivalent circuit shown in Fig. 1 are then fitted to give a best match with the measured parameters [27-29]. However, for a given voltage amplitude the capacitance measurements with two frequency methods have a lower signal-to-noise ratio than the sine plus dc technique [12].

\subsubsection{Square wave methods}

An alternative approach utilizes the measurement and analysis of current transients produced by square pulses. For the equivalent circuit of Fig. 1 the current transients can be fitted with a single exponential providing three measured quantities, the amplitude of the capacitive current transient, its time constant, and the steady state current. The method allows determination of the three equivalent circuit parameters without requiring knowledge of $V_{\text {rev }}$ [22] and is implemented in the Patchmaster software to perform automatic whole cell capacitance compensation and capacitance tracking. This technique was typically used with a low duty cycle but an improved signal-to-noise ratio is obtained when a continuous square wave is used. The currents produced by a periodic square wave may not only be analyzed by exponential fitting but can also be processed with a lock-in amplifier type algorithm. A continuous square wave can be represented as a Fourier sum of square waves where the lowest frequency that equals the square wave frequency has the highest amplitude. Indeed, the amplitude of this component exceeds the amplitude of the square wave by a factor 1.27 [30]. When a square wave is used in the Patchmaster software instead of the commonly used sine wave with the same peak amplitude, the signal-to-noise ratio is correspondingly increased (Herbst and Lindau, unpublished). If the raw currents are stored, the method has the advantage that they can be analyzed offline by single exponential fits to determine if the equivalent circuit of Fig. 1 is valid and if the assumption for $V_{\text {rev }}$ can be confirmed or needs to be modified. This is particularly valuable for experimental conditions where large changes in $\mathrm{R}_{\mathrm{m}}$ occur.

\subsubsection{Stimulation of exocytosis using voltage clamp pulses}

In excitable cells, such as neurons, chromaffin cells and beta cells, the principal stimulus of secretion is $\mathrm{Ca}^{2+}$ entry through voltagegated calcium channels. Experimentally, a widely used approach is to stimulate exocytosis in whole cell patch clamp experiments under voltage clamp by applying depolarizing pulses [21,31-34]. In such experiments exocytosis stimulated by the pulse can be recorded by capacitance measurements. Typically, the capacitance measurement is interrupted while the depolarization pulse is applied for two reasons:

The first reason is that when the sine wave or square wave stimulation used to measure capacitance is suspended, it is possible to measure the current that is activated by the depolarization pulse. If the compositions of the solutions are properly chosen, minimizing contributions from $\mathrm{Na}^{+}$currents and $\mathrm{K}^{+}$currents, the $\mathrm{Ca}^{2+}$ current during the pulse can be determined and the $\mathrm{Ca}^{2+}$ influx calculated $[31,33,35]$. Although this does not directly provide a measure of the intracellular $\mathrm{Ca}^{2+}$ concentration change, it allows comparison of $\mathrm{Ca}^{2+}$ influx in different stimulations to ensure for instance that different stimuli in a particular experiment produced similar $\mathrm{Ca}^{2+}$ currents and that changes in exocytosis are not a consequence of changes in $\mathrm{Ca}^{2+}$ influx.

A second reason is that the $\mathrm{Ca}^{2+}$ measurement during the depolarization pulse may be incorrect due to the activation of voltagegated ion channels. It must be kept in mind that the equivalent circuit of Fig. 1 is based on the assumption that the membrane conductance is independent of voltage in the voltage range of the command signal that is used to measure capacitance. Typically, the command voltage range is $20-50 \mathrm{mV}$ peak-to-peak. At a sufficiently negative holding potential this will not lead to significant activation of voltage-gated ion channels. However, during a stimulating depolarization pulse there is a marked activation of voltage-gated ion channels affecting the capacitance measurement. This generates a complex current and leads to artifacts in the capacitance measurement [36]. The apparent changes in the capacitance trace are related to the nonlinearity of the activated conductances and in particular to the finite kinetics with which they respond to changes in membrane potential, which produce phase-shifted currents [36]. The artifacts in the capacitance measurements are most pronounced when the angular frequency of the sine wave is close to the value of the rate constant of the channel. However, when the channel kinetics are much slower or much faster the artifacts are smaller and their effects on the capacitance measurements may be estimated, if the kinetics and voltage dependence of the activated currents are known [36]. Nevertheless, this has only been done for stimulation with very long pulses having a duration of several seconds [37] und it seems unlikely that the time course of a capacitance change during a depolarization pulse can be extracted on the millisecond time scale $[24,36]$.

\subsubsection{Pulse protocols for determination of the readily releasable pool}

Early experiments where exocytosis was stimulated with pulse trains or with pulses of long duration had suggested that a limited pool of readily releasable vesicles exists (RRP) that generates a rapid increase in capacitance but that is also rapidly exhausted $[33,37,38]$. To quantify the RRP size a method that is now widely used employs a double pulse protocol [31]. Two depolarization pulses of equal duration are given, separated by a relatively short time interval, for instance two $100 \mathrm{~ms}$ pulses separated by a $100 \mathrm{~ms}$ inter-pulse interval [31] The pulse duration must be long enough such that the first pulse produces significant depletion of the readily releasable pool $\left(\Delta C_{R R P}\right)$ and the capacitance increase induced by the second pulse $\left(\Delta \mathrm{C}_{\mathrm{M}, 2}\right)$ is significantly smaller than that produced by the first pulse $\left(\Delta C_{M, 1}\right)$. Therefore, the pulse durations may need to be longer than $100 \mathrm{~ms}$ depending on cell type and experimental conditions. The estimate of the RRP is based on the assumption that the first pulse induces exocytosis of a certain fraction $f$ of the RRP and that the second pulse induces exocytosis of the same fraction but from what is now left from the RRP [31]:

$$
\begin{aligned}
& \Delta C_{M, 1}=f \cdot C_{R R P} \\
& \Delta C_{M, 2}=f \cdot\left(C_{R R P}-\Delta C_{M, 1}\right)
\end{aligned}
$$

The RRP can thus be estimated as

$C_{R R P}=\frac{\Delta C_{M, 1}+\Delta C_{M, 2}}{1-\left(\frac{\Delta C_{M, 2}}{\Delta C_{M, 1}}\right)^{2}}$

which is equivalent to

$$
\Delta C_{R R P}=\frac{\Delta C_{M, 1}}{1-\frac{\Delta C_{M, 2}}{\Delta C_{M, 1}}}
$$

Clearly, the inter-pulse interval must be short enough such that no significant refilling of the RRP occurs. This can be tested by varying the length of the inter-pulse interval duration, which can thereby reveal the time course of refilling the RRP [39].

\subsubsection{Stimulation of exocytosis using caged calcium}

While capacitance measurements in combination with pulse stimulation and measurements of calcium currents make it possible to relate the exocytotic response to calcium influx, it does not provide a good estimate of the actual free intracellular $\mathrm{Ca}^{2+}$ concentration 
$\left(\left[\mathrm{Ca}^{2+}\right]_{\mathrm{i}}\right)$. The reason is that with $\mathrm{Ca}^{2+}$ influx through channels in the plasma membrane, much of the $\mathrm{Ca}^{2+}$ entering the cell will be bound by endogenous $\mathrm{Ca}^{2+}$ binding sites or exogenous $\mathrm{Ca}^{2+}$ buffers of the pipette solution, taken up into organelles, or pumped out through the plasma membrane [40]. One can, in addition to measuring $\mathrm{Ca}^{2+}$ currents, include a fluorescent $\mathrm{Ca}^{2+}$ indicator such as fura- 2 to measure the change in $\left[\mathrm{Ca}^{2+}\right]_{\mathrm{i}}$ but this does still not provide the $\mathrm{Ca}^{2+}$ concentration at the site of fusion because of steep $\left[\mathrm{Ca}^{2+}\right]$ gradients near the plasma membrane [40-42] and micro- or nanodomains in the vicinity of individual calcium channels [43-45].

To avoid these difficulties, photorelease of caged calcium has been employed as a stimulus in whole cell patch clamp experiments, which produces a homogeneous step increase of $\left[\mathrm{Ca}^{2+}\right]_{i}[46-48]$. The preferred caged $\mathrm{Ca}^{2+}$ compound currently used is nitrophenyl-EGTA (NP-EGTA), which absorbs light below $400 \mathrm{~nm}$ wavelength with a peak near $260 \mathrm{~nm}$ [49]. Upon photolysis with a brief laser flash, NPEGTA saturated with $\mathrm{Ca}^{2+}$ is rapidly photolyzed and releases $\mathrm{Ca}^{2+}$ with a time constant $<15 \mu$ s [50].

For measurements of $\mathrm{Ca}^{2+}$ activated exocytosis, whole cell capacitance measurements are performed with a pipette solution containing $\sim 5$ mM NP-EGTA, 3.5-4 $\mathrm{mM} \mathrm{Ca}^{2+}$ as well as two ratiometric fluorescent $\mathrm{Ca}^{2+}$ indicator dyes with different affinities $[51,52]$. Typically, a mixture of $0.4 \mathrm{mM}$ Fura-4F and $0.4 \mathrm{mM}$ Furaptra is now used [52]. The fluorescence ratio of these dyes, measured with excitation alternating between two appropriate excitation wavelengths (e.g. $350 / 380 \mathrm{~nm}$ ), is calibrated with buffered solutions in living cells [51]. The use of the two-dye mixture allows determination of pre-flash as well as post-flash $\left[\mathrm{Ca}^{2+}\right]_{\mathrm{i}}$ in a wide concentration range. Photolysis is in most experiments produced by a flash lamp (Rapp Optoelektronik), which is directed onto the cell via the epifluorescence port of the microscope using a dual port condenser (Till photonics), which allows combination with the fluorescence excitation from a monochromator (Till photonics). The strong flash that photolyzes NP-EGTA may also produce some photodamage of the $\mathrm{Ca}^{2+}$ indicator dyes, which may lead to a post-flash change in the indicator calibration [48]. It has recently been reported that the photodamage can be reduced by including $1 \mathrm{mM}$ ascorbic acid in the pipette solution, which protects the $\mathrm{Ca}^{2+}$ indicator dyes [52] such that the calibration of the fluorescence ratio remains unchanged after the flash.

Exocytosis stimulated by photorelease of caged $\mathrm{Ca}^{2+}$ can be recorded by whole cell capacitance measurements, which are typically performed using the sine $+\mathrm{dc}$ method. Since no depolarization pulse is used, the holding potential is kept at $\sim-70 \mathrm{mV}$ and therefore the capacitance measurement can be continuous. In chromaffin cells, carbon fiber amperometry can simultaneously be used as an additional monitor of catecholamine release. This can aid in identifying if the observed capacitance change reflects exocytosis of catecholamine containing chromaffin granules. It has been reported that at excessively high $\left[\mathrm{Ca}^{2+}\right]_{\mathrm{i}}$ large capacitance changes may occur that are not mediated by the neuronal SNARE complex [53] and are not associated with catecholamine release measured amperometrically [54].

The flash intensity is typically chosen to produce a step change of $\left[\mathrm{Ca}^{2+}\right]_{\mathrm{i}}$ to $\sim 20 \mu \mathrm{M}$ [55], a concentration that may be experienced with physiological stimulation by vesicles that are relatively close to $\mathrm{Ca}^{2+}$ channel. Following a step increase of $\left[\mathrm{Ca}^{2+}\right]_{\mathrm{i}}$ to $\sim 20 \mu \mathrm{M}$ exocytosis begins with a rapid exocytotic burst with millisecond kinetics followed by a slower, more sustained phase on the time scale of seconds [46-48]. This observation of an exocytotic burst in response to a step change in $\left[\mathrm{Ca}^{2+}\right]_{\mathrm{i}}$ provided independent confirmation of the earlier conclusions derived from experiments with pulse depolarization described above. The basal pre-flash $\left[\mathrm{Ca}^{2+}\right]_{i}$ affects the priming of vesicles and it was found that the RRP is maximal around $\left[\mathrm{Ca}^{2+}\right]_{\mathrm{i}}=500 \mathrm{nM}$. At lower $\left[\mathrm{Ca}^{2+}\right]_{i}$ priming decreases due to a decreased priming rate, at higher $\left[\mathrm{Ca}^{2+}\right]_{\mathrm{i}}$ priming decreases due to increased stimulation of fusion beginning to deplete the RRP [51].

The exocytotic burst itself can be fitted by a double exponential time course [55] and the fast component of the burst appeared to be equivalent to the RRP observed with the double pulse stimulation protocol described above [51]. The slow component of the burst has accordingly been named slowly releasable pool (SRP). The time constants of the two components reflecting release from RRP and SRP show a steep $\left[\mathrm{Ca}^{2+}\right]_{\mathrm{i}}$ dependence but their amplitudes do not. These results led to the conclusion that the $\left[\mathrm{Ca}^{2+}\right]_{i}$ dependence of transmitter release in response to action potentials or brief depolarizations is a consequence of the $\left[\mathrm{Ca}^{2+}\right]_{\mathrm{i}}$ dependence of the release kinetics, which converts to a $\left[\mathrm{Ca}^{2+}\right]$ dependence of the release amplitude when $\left[\mathrm{Ca}^{2+}\right]_{\mathrm{i}}$ is elevated transiently [55]. The high time resolution of the capacitance measurements in conjunction with flash release of caged calcium has enabled an enormous body of work investigating the molecular steps of priming and fusion, which is beyond the scope of this review. The combination of such experiments with molecular and pharmacological manipulations has opened a wide field of experiments providing direct information on the regulation of vesicle pool sizes and their release.

\subsubsection{Single fusion pore recording}

Key steps in exocytosis are the formation and the expansion of the fusion pore that forms the connection between the vesicular lumen and the extracellular space and that allows for transmitter release. The measurements of biophysical fusion pore properties provide insight into the function and structure of the fusion pore analogous to that obtained from single channel recordings for ion channels. Capacitance measurements can be utilized to obtain a time-resolved recording of single fusion pore conductance. In the state where a narrow fusion pore forms, the equivalent circuit of Fig. 1 cannot be used because the charging of the vesicle membrane $C_{V}$ via a fusion pore with low conductance $G_{P}$ (or high resistance $R_{P}$ ) produces a time constant that may be much longer than that of charging $C_{M}$ via the access resistance $R_{A}$. The equivalent circuit for such a state is shown in Fig. 2.

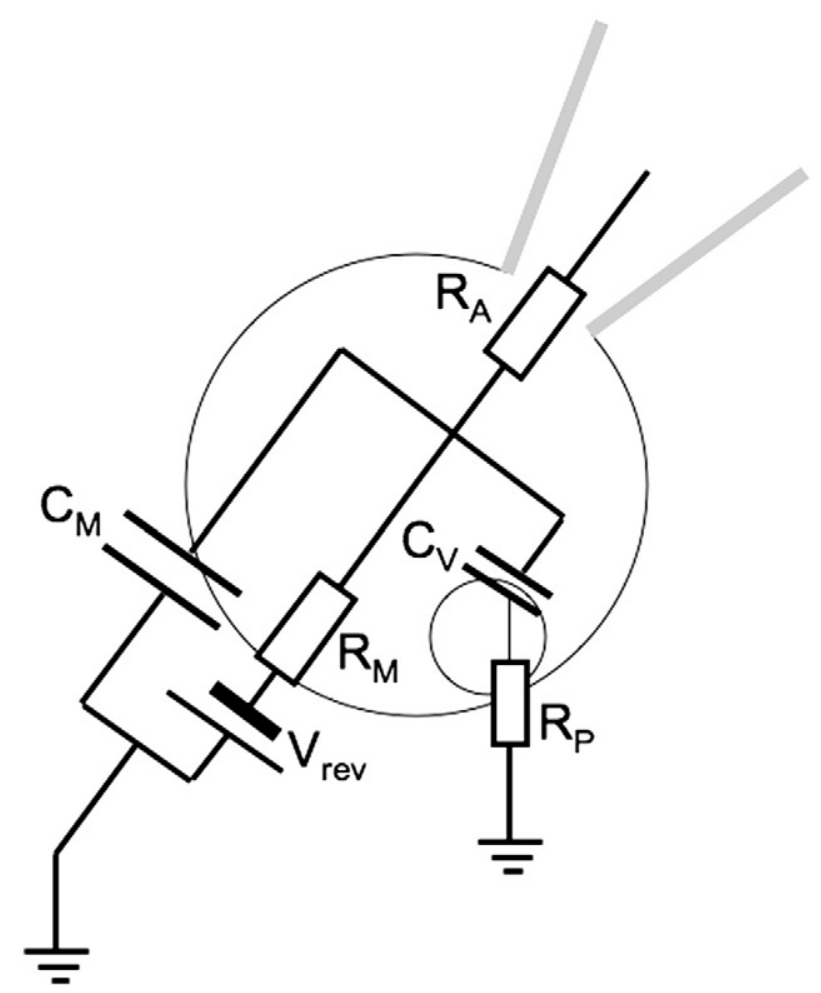

Fig. 2. Whole cell equivalent circuit of a cell with a vesicle of capacitance $C_{V}$ connected to extracellular space via a narrow fusion pore with conductance $G_{P}=1 / R_{P}$. The other elements of the equivalent circuit are as in Fig. 1. 
2.1.8. Fusion pore measurements using the piecewise linear technique

Clearly, using a single sine wave or even dual sine wave does not allow for the determination of the five parameters of this equivalent circuit. Measurements of fusion pore properties were therefore performed using either the piecewise linear technique or using time domain analysis of currents evoked by square voltage pulses. In the whole cell configuration fusion of single vesicles and determination of fusion pore conductance is only possible for cells with relatively large secretory granules. When the piecewise linear technique is used, the fusion of a single vesicle with capacitance $C_{V}$ having a fusion pore with conductance $G_{P}$ produces the changes [56-58]

$\Delta Y_{C}=T^{2} \cdot \frac{\omega C_{V}}{1+\left(\omega C_{V} / G_{P}\right)^{2}}$

$\Delta Y_{R}=T^{2} \cdot \frac{\left(\omega C_{V}\right)^{2} / G_{P}}{1+\left(\omega C_{V} / G_{P}\right)^{2}}$

Vesicle capacitance and fusion pore conductance can be calculated from these measured changes of the lock-in amplifier outputs as [23,59]

$C_{V}=\frac{1}{\omega T^{2}} \frac{\left(\Delta Y_{R}\right)^{2}+\left(\Delta Y_{C}\right)^{2}}{\Delta Y_{C}}$

$G_{P}=\frac{1}{T^{2}} \frac{\left(\Delta Y_{R}\right)^{2}+\left(\Delta Y_{C}\right)^{2}}{\Delta Y_{R}}$

\subsubsection{Fusion pore measurements using charging current transients}

Determination of fusion pore properties from capacitance measurements typically has a time resolution of $\sim 10 \mathrm{~ms}$. However, submillisecond time resolution can be achieved for very large vesicles when capacitance measurements are performed in a discontinuous manner such that the membrane current can be measured with high time resolution at constant voltage between the capacitance measurement [56]. When a fusion pore opens with an initial conductance $G_{P}$ in a voltage clamp whole cell recording, the vesicle membrane is charged to the holding potential of the plasma membrane and this charging current flows through the fusion pore. The time integral of this transient fusion pore current $I_{P}$ provides the total charge $Q_{P}$ of this process. Since the vesicle capacitance is known from the difference of the membrane capacitance before and after the fusion event, the initial potential difference between plasma membrane and vesicle membrane can be determined as

$V_{P}(t=0)=\frac{Q_{P}}{C_{V}}$

and the initial fusion pore conductance is obtained from the fusion pore current amplitude as

$G_{P}=\frac{I_{P}}{V_{P}}$

If $G_{P}$ is constant, the fusion pore current $I_{P}$ decays exponentially with a time constant

$\tau=\frac{C_{V}}{G_{P}}$

Fusion of a vesicle with $100 \mathrm{fF}$ capacitance and a typical fusion pore conductance of $350 \mathrm{pS}$ would produce a transient fusion pore current with a decay time constant of $\sim 300 \mu$ s.

\subsubsection{Fusion pore measurements using square pulses}

For cells with such large vesicles capacitance measurements with square voltage pulses can provide additional information. When a voltage step is applied to a cell represented by the equivalent circuit of Fig. 2, the resulting current will show a double exponential decay providing 5 parameters: The amplitudes and time constants of the two exponentials and the steady state baseline current, which is sufficient to determine all 5 parameters of the equivalent circuit [60]. The method has revealed detailed insight into the role of $\left[\mathrm{Ca}^{2+}\right]_{\mathrm{i}}$ in the differential regulation of exocytosis, vesicle-vesicle fusion and compound exocytosis [61-64].

\subsection{Patch capacitance measurements to record fusion of small vesicles}

As mentioned before, the noise of capacitance measurements increases with $C_{M}$ and cells with smaller capacitance give recordings with lower noise. In most cell types that undergo calcium-stimulated exocytosis, such as nerve terminals, chromaffin cells or other neuroendocrine cells, secretory vesicles are small, which makes it very difficult to detect individual fusion events in the whole cell configuration, even when small cells are chosen. As for single channel recordings [20], much lower noise is obtained in capacitance measurements from cell-attached or excised patches, which allows observation of single exocytosis and endocytosis events as discrete capacitance steps [21]. The equivalent circuit for the cell-attached configuration is shown in Fig. 3. The capacitance of the patch $C_{P}$ is in series with $C_{M}$ but $C_{M}$ is much greater than $C_{P}$ and the total capacitance is thus approximately equal to $C_{P}$

Patch capacitance measurements are generally performed using the piecewise linear technique and typically a sine wave frequency of $\sim 20 \mathrm{kHz}$ is now used where the capacitance noise approaches a minimum [65]. The method has been implemented in the Patchmaster lock-in extension software (HEKA Elektronik) [66]. Depending on the sine wave amplitude used, the noise level in such measurements

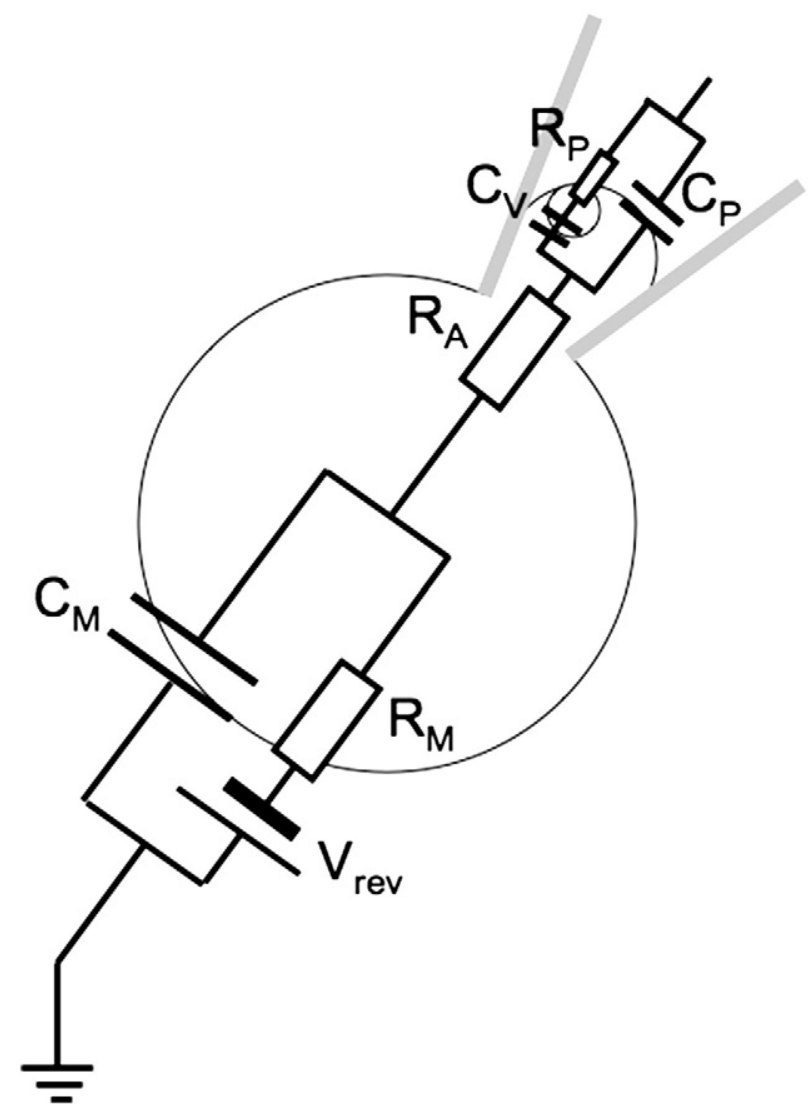

Fig. 3. Equivalent circuit for cell-attached patch capacitance measurements. $C_{P}$ is the capacitance of the membrane patch in the pipette tip. The other elements of the equivalent circuit are as in Fig. 2. 
is reduced to $0.025 \mathrm{fF}$ or less such that capacitance steps of $0.1 \mathrm{fF}$, corresponding to exo- and endocytosis of single $60 \mathrm{~nm}$ vesicles, can be clearly resolved [59]. Due to the high frequency, larger amplitudes can be used, typically $50 \mathrm{mV}$ rms, which corresponds to $141 \mathrm{mV}$ peakto-peak. The advantage of using a high sine wave frequency is not only the low capacitance measurement noise but also the fact that the angular frequency $\left(1.25 \times 10^{5} \mathrm{~s}^{-1}\right.$ for a $20 \mathrm{kHz}$ sine wave $)$ is much faster than the gating rate constants of voltage-gated ion channels, such that the sine wave has little effect on channel gating and artifacts in the capacitance measurement are minimal [36]. In some experiments even $200 \mathrm{mV}$ rms amplitude has been used [67] although we found that this is often detrimental to the stability of the seal resistance. Also, use of high amplifier gain for low noise is practically impossible with such high amplitude because capacitance compensation is extremely critical to avoid saturation of the amplifier.

Patch capacitance measurements allow the direct measurement of capacitance steps sizes, which are generally consistent with the size distribution of secretory vesicles [68]. However, the observation of very large capacitance steps under conditions of enhanced $\left[\mathrm{Ca}^{2+}\right]_{\mathrm{i}}$ elevation has provided direct evidence for compound exocytosis in nerve terminals [67]. A unique aspect of capacitance measurements is that not only the fusion event leading to release can be detected but also the subsequent re-uptake by endocytosis, which manifests itself as a downward (negative) capacitance step. Transient fusion events, also named 'kiss-and-run' events can be detected as capacitance transients or 'flickers' and have been observed in various cell types and nerve terminals undergoing calcium-activated exocytosis [69-76].

Fig. 4A shows a photograph of a bovine chromaffin cell patched in the cell-attached configuration and a section of the recording from this cell is shown in Fig. 4B. The capacitance trace (Fig. 4B, lower trace) shows two full fusion events (labeled $\mathrm{c}$ and e) as well as a transient fusion event between them (labeled d). The transient increases in the conductance trace (Fig. 4D, upper trace) are due to a low fusion pore conductance and will be discussed in more detail in Section 2.2.2.

\subsubsection{The phase problem of patch capacitance measurements}

A difficulty with the patch capacitance measurements is the correct determination of the phase where changes in patch capacitance appear only in the corresponding output of the lock-in amplifier and have no projection into the conductance trace. In whole cell recordings, the phase can be determined by applying a small change to the whole cell capacitance compensation (C slow). If the compensation is set correctly, the phase will be equally correct for capacitance changes of the cell membrane. In patch capacitance measurements the situation is different because the capacitance of the pipette tip immersed in the bath, the capacitance of the patch, and other stray capacitances are all lumped together and are compensated together using the $\mathrm{C}$ fast compensation. The phase for capacitance changes in the patch is, however, determined only by the patch time constant, analogous to the phase setting of a whole cell recording [65]. In the cell-attached configuration the time constant of the $\mathrm{C}$ fast compensation is therefore not identical with the time constant of the patch and $\mathrm{C}$ fast dithering gives only an approximate phase setting. One would normally use $C$ fast capacitance dithering and adjust the phase of the lock-in amplifier such that a change appears only in the capacitance trace with no change in the conductance trace. This phase will, however, not be exactly correct for the time constant of the patch. One way to generate a pure change in $C_{P}$ is to apply some suction to the patch pipette, which produces transient capacitance changes as more membrane is pulled into the pipette while the extra suction is applied [65]. The phase can then be adjusted such that the changes have no projection into the conductance trace. With this method, the
A

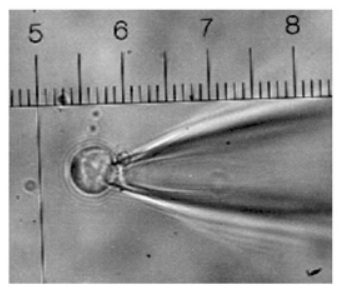

C
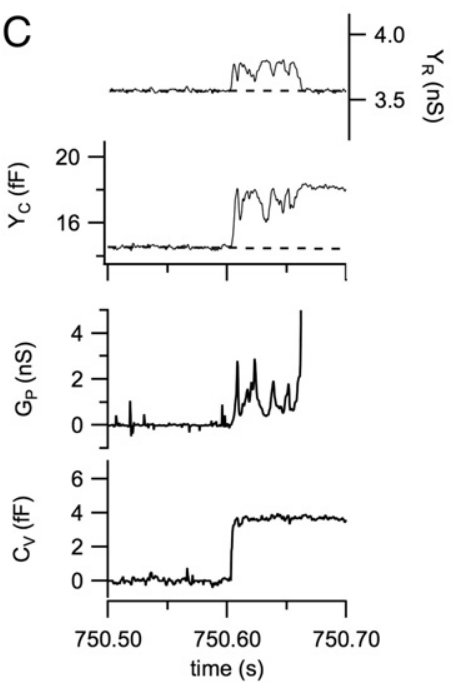

B

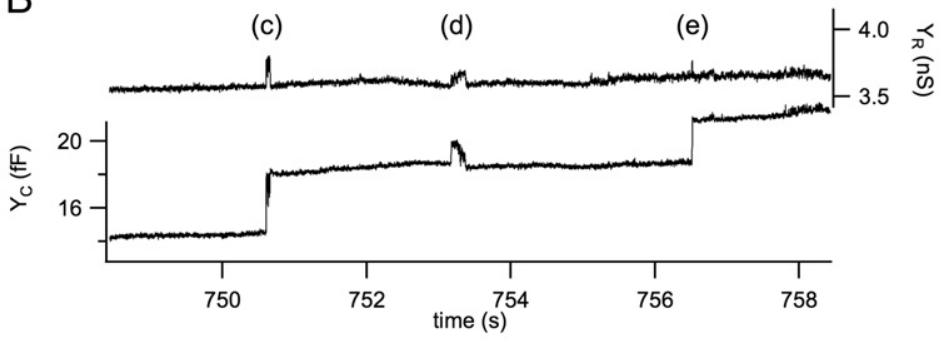

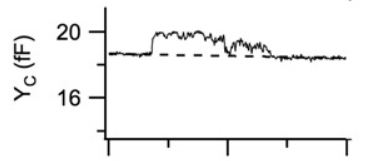
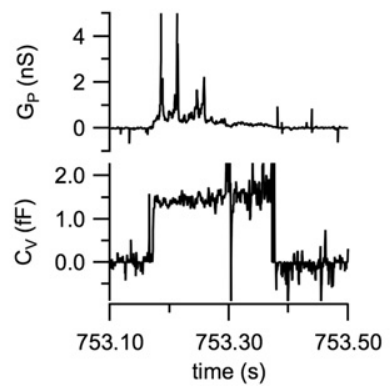

E
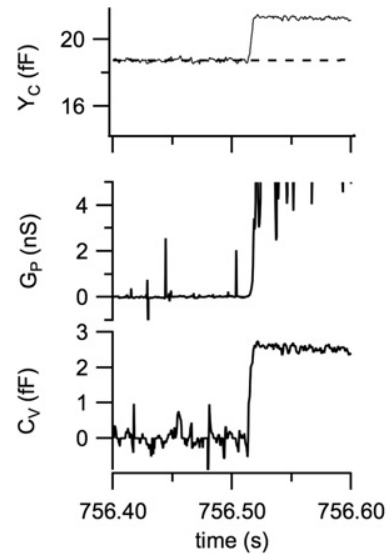

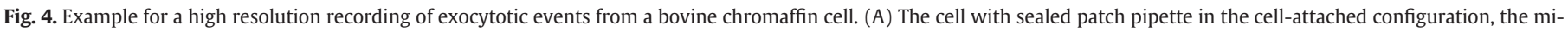

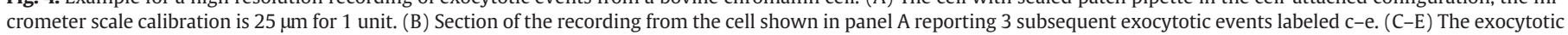
events labeled c-e in panel $B$ and their analysis on expanded time scales; traces from top to bottom are $Y_{R}, Y_{C}, G_{P}$, and $C_{V}$. 
adjustment of the lock-in amplifier phase setting can be done during the experiment. However, the correct phase setting may change during an experiment due to changes in $C_{P}$ and $R_{A}$. It is therefore often more practical to perform the recording at some fixed approximate phase setting and adjust the phase offline during the analysis.

A phase error $\Delta \varphi$ will manifest itself as a projection such that a capacitance step is accompanied by a step in the conductance trace and a conductance change will be accompanied by a corresponding change in the capacitance trace. To correct the phase error, phase-shifted traces $Y_{C}$ and $Y_{G}$ can be calculated from the originally recorded traces $\mathrm{Y}_{\mathrm{C}}^{0}$ and $\mathrm{Y}_{\mathrm{G}}^{0}$ as

$Y_{C}=Y_{C}^{0} \cdot \cos (\Delta \varphi)+Y_{G}^{0} \cdot \sin (\Delta \varphi)$

$Y_{G}=Y_{G}^{0} \cdot \cos (\Delta \varphi)-Y_{C}^{0} \sin (\Delta \varphi)$

$[23,77]$. If patch capacitance changes were produced during the recording by applying transient suction to the pipette, the phase shift can be calculated from the deflections in the capacitance and conductance traces [65] as

$\Delta \varphi=\tan ^{-1}\left(\frac{\Delta Y_{G}^{0}}{\Delta Y_{C}^{0}}\right)$

In practice, one may try different values for the phase until the projection in the conductance trace vanishes. In the absence of suctioninduced pure capacitance changes, capacitance steps can be used to find a phase were the projection in the conductance trace is absent or conductance changes, such as channel openings, can be identified and their projection in the capacitance trace eliminated by phase adjustment. Single channel openings can be recorded in the conductance trace because the $20 \mathrm{kHz}$ sine wave frequency is much faster than the channel gating. At this frequency opening of a channel with $20 \mathrm{pS}$ conductance generates a deflection in the conductance trace with the same amplitude as a $0.16 \mathrm{fF}$ step in the capacitance trace. Fig. 4D shows no steps in the conductance trace associated with the capacitance steps such that the conductance trace baseline before and after a capacitance step is unchanged, which indicates correct phase setting.

The high sine wave frequency also makes it possible to record, in addition to the conductance and capacitance trace, also the membrane current during the capacitance measurement. Due to the usually imperfect capacitance compensation the membrane current is dominated by the $20 \mathrm{kHz}$ sine wave. However, this contribution can be easily removed by low-pass filtering at $3 \mathrm{kHz}$ or below such that changes in membrane current can be recorded simultaneously [78].

\subsubsection{Fusion pore measurements in small vesicles}

In patch capacitance measurements the piecewise linear technique allows determination of fusion pore conductance as in whole cell recordings using Eqs. (9) and (10) [59]. For such an analysis it is particularly important that the phase is set to the correct value. Under most conditions, vesicles will fuse with the plasma membrane in a way such that the fusion pore conductance eventually becomes very large such that the capacitance trace shows the full value of $C_{V}$ and the capacitance trace shows no change. While the fusion pore has a small conductance, there is a transient signal $\Delta \mathrm{Y}_{\mathrm{R}}$ and a reduced signal $\Delta \mathrm{Y}_{\mathrm{C}}$ according to Eqs. (7) and (8). An example is the event (c) of Fig. 4B, which is shown on expanded time scale in Fig. 4C. The transient increase in the conductance trace (Fig. $4 \mathrm{C}$, top trace) is accompanied by fluctuations in the capacitance trace (Fig. 4C, 2nd trace). At the time where the transient in the conductance trace ends, the conductance trace attains its final value indicating the capacitance increase produced by this vesicle, in this case $3.6 \mathrm{fF}$. Using the deviations in the capacitance and conductance trace from their respective baselines (dashed) the time course of fusion pore conductance can be calculated according to Eq. (10) (Fig. 4C, 3rd trace). The vesicle capacitance calculated according to Eq. (9) is constant during the fusion pore opening (Fig. 4C, bottom trace) which confirms the interpretation of the observed fluctuations as a consequence of fusion pore conductance fluctuations. The $2.6 \mathrm{fF}$ capacitance step labeled (e) is shown on expanded time scale in Fig. 4E. This event also shows a transient increase in conductance trace associated with the capacitance step but this transient has a very short duration that is in fact limited by the low-pass filter of the lock-in amplifier. Such events can therefore not be used to determine a reliable value for the fusion pore conductance. Typically we choose to define the value of the initial fusion pore conductance as the $G_{P}$ value at the time where the increase in the $C_{V}$ trace has reached a value $>80 \%$ of the final $C_{V}$ value. In the case of Fig. $4 C G_{p}$ initial is $\sim 320 \pm 100 \mathrm{pS}$. The fusion pore makes several attempts to open to a large size but frequently returns to values in the 400-500 pS range until it finally opens fully (Fig. 4C, 3rd trace).

If the conductance trace shows a change relative to the pre-fusion baseline after the fusion pore has expanded, the phase may be incorrect. However, the possibility that a true conductance change occurs associated with fusion cannot be excluded. To determine if the change in the conductance trace reflects a true change in patch membrane conductance and not a projection from the capacitance change due to incorrect phase setting, the measurement of the low-pass filtered membrane current is helpful because a change in membrane conductance is likely associated with a measurable change in current.

The determination of the correct phase for a sequence of capacitance steps associated with full fusion is usually straightforward by minimizing the projection in the conductance trace as shown in Fig. 4B. Determination of fusion pore conductance for transient fusion pore openings where the capacitance trace shows transient changes are more problematic. If the transient capacitance flicker is associated with a deflection in the conductance trace, it may be that the fusion pore that opens transiently has a small conductance that can then be calculated using Eq. (9). Event (d) in Fig. 4B is such an example and the analysis is shown in Fig. 4D. The initial fusion pore conductance for this event was $295 \pm 15 \mathrm{pS}$ and the $\mathrm{G}_{\mathrm{p}}$ time course shows again a few attempts to open fully but mostly fluctuates in a range of 100-600 pS. The correctness of the fusion pore analysis is confirmed by the constant $C_{V}$ of $\sim 1.5 \mathrm{fF}$ during this transient fusion event (Fig. 4D, bottom trace), which does not lead to full fusion.

The recording of Fig. 4B shows clear capacitance steps close in time to the capacitance flicker and since these capacitance steps show no projection in the conductance trace, one can be confident that the flickers are also recorded at the correct phase and the fusion pore analysis is valid. However, If capacitance flickers indicating kiss-and-run events are not intermingled with full fusion capacitance steps, correlated changes in conductance trace and capacitance trace may simply be fully or in part due to an error in the phase setting, such that the conductance trace simply reflects a projection of the capacitance change. Such events should be viewed with caution. If a trace shows only capacitance flickers with associated flickers in the conductance trace it may be almost impossible to determine the correct phase for the respective section of the recording. The reason is that the phase of the patch can change. The phase depends on the patch time constant

$$
\tau_{P}=R_{A} \cdot C_{P}
$$

and organelles that may partially obstruct the pipette tip may change their position. Furthermore, patch area may change due to suction imbalance leading to baseline drift or due to exocytosis and endocytosis. Determination of fusion pore conductance from standalone capacitance flickers may therefore not always be correct.

\subsubsection{Patch amperometry}

In some experiments with chromaffin cells, patch capacitance measurements were combined with amperometric detection of 
catecholamine release by inserting a carbon fiber inside the patch pipette using a specially designed pipette holder, a method that was named patch amperometry $[68,77,78]$. With the carbon fiber inside the patch pipette, the regular patch pipette electrode must be connected as ground electrode and the sine wave to measure capacitance applied to the bath electrode. The method has the advantage that the capacitance measurement is combined with amperometric detection of release. The carbon fiber measures individual release events as amperometric current spikes and the integrated charge of these spikes provides the number of molecules that were released from a vesicle. It is thereby possible to relate the amount of molecules released from a particular vesicle to the size of that vesicle, which can be estimated from the capacitance step size $[68,79,80]$. In this way the vesicular concentration can be determined directly.

Patch amperometry recordings in chromaffin cells have shown directly that the amperometric foot current, which is often seen preceding an amperometric spike, reflects the slow leakage of catecholamines through a narrow fusion pore [68], as previously observed in mast cells combining whole cell capacitance measurements with conventional amperometry [81]. The measurements showed directly that the amperometric foot current indicating the rate of catecholamine release from chromaffin granules fluctuates synchronously with fluctuations in fusion pore conductance [82]. The fusion pore thus represents the diffusion barrier for catecholamine release. Since the catecholamine molecules are charged, the release through a narrow fusion pore reflects an electrodiffusion process that involves charge compensation by entry of sodium ions through the fusion pore from the extracellular medium [82].

\section{Conclusions}

Calcium-activated exocytotic fusion of secretory vesicles with the plasma membrane increases the plasma membrane area, which leads to a proportional increase in plasma membrane capacitance. In contrast to other methods measuring transmitter release, capacitance measurements therefore provide a direct measurement of exocytotic fusion. Capacitance measurements can provide detailed information on the extent and kinetics fusion as well as detailed aspects of the mode of fusion and enables distinction of single vesicle full fusion, transient kiss-and-run fusion or multivesicular compound exocytosis. Furthermore, measurement of fusion pore conductances and their dynamic behavior has enabled the characterization fusion pore properties in way that resembles the characterization of ion channel function through single channel recordings. The combination of patch clamp capacitance measurements with pharmacological and molecular manipulations of exocytosis will continue to provide further insight into the molecular mechanisms of calcium-activated exocytotic fusion pore formation.

\section{Acknowledgements}

I am indebted to Dr. Qinghua Fang for the experiment shown in Fig. 4. This work has been supported by NIH grant R01GM085808.

\section{References}

[1] M. Lindau, G. Alvarez de Toledo, The fusion pore, Biochim. Biophys. Acta 1641 (2003) 167-173.

[2] B. Katz, R. Miledi, The effect of temperature on the synaptic delay at the neuromuscular junction, J. Physiol. 181 (1965) 656-670.

[3] B. Katz, R. Miledi, The measurement of synaptic delay, and the time course of acetylcholine release at the neuromuscular junction, Proc. R. Soc. London, B. Biol. Sci. 161 (1965) 483-495.

[4] R. Llinas, I.Z. Steinberg, K. Walton, Relationship between presynaptic calcium current and postsynaptic potential in squid giant synapse, Biophys. J. 33 (1981) 323-351.

[5] P. Fatt, B. Katz, Some observations on biological noise, Nature 166 (1950) 597-598.
[6] E.D. De Robertis, H.S. Bennett, Some features of the submicroscopic morphology of synapses in frog and earthworm, J. Biophys. Biochem. Cytol. 1 (1955) 47-58.

[7] J. Del Castillo, B. Katz, La base "quantale" de la transmission neuromusculaire, Colloques Internat, C.N.R.S. 67 (1957) 245-256.

[8] F.A. Edwards, A. Konnerth, B. Sakmann, Quantal analysis of inhibitory synaptic transmission in the dentate gyrus of rat hippocampal slices: a patch-clamp study, J. Physiol. 430 (1990) 213-249.

[9] Z.W. Wang, Origin of quantal size variation and high-frequency miniature postsynaptic currents at the Caenorhabditis elegans neuromuscular junction, J. Neurosci. Res. 88 (2010) 3425-3432.

[10] R.M. Wightman, J.A. Jankowski, R.T. Kennedy, D.T. Kawagoe, T.J. Schroeder, D.J. Leszczyszyn, J.A. Near, E.J. Diliberto Jr., O.H. Viveros, Temporally resolved catecholamine spikes correspond to single vesicle release from individual chromaffin cells, Proc. Natl. Acad. Sci. U. S. A., 88, 1991, pp. 10754-10758.

[11] R.H. Chow, L.V. Rüden, E. Neher, Delay in vesicle fusion revealed by electrochemical monitoring of single secretory events in adrenal chromaffin cells, Nature 356 (1992) 60-63.

[12] R. Borges, M. Camacho, K.D. Gillis, Measuring secretion in chromaffin cells using electrophysiological and electrochemical methods, Acta Physiol. 192 (2008) 173-184 (Oxf).

[13] L. Mellander, A.S. Cans, A.G. Ewing, Electrochemical probes for detection and analysis of exocytosis and vesicles, Chemphyschem 11 (2010) 2756-2763.

[14] E.V. Mosharov, D. Sulzer, Analysis of exocytotic events recorded by amperometry, Nat. Methods 2 (2005) 651-658.

[15] K.S. Cole, Electric impedance of Hipponoë eggs, J. Gen. Physiol. 18 (1935) 877-887.

[16] B.A. Afzelius, The ultrastructure of the cortical granules and their products in the sea urchin egg as studied with the electron microscope, Exp. Cell Res. 10 (1956) 257-285.

[17] Rothschild, The membrane capacitance of the sea urchin egg, J. Biophys. Biochem. Cytol. 3 (1957) 103-110.

[18] L.A. Jaffe, S. Hagiwara, R.T. Kado, The time course of cortical vesicle fusion in sea urchin eggs observed as membrane capacitance changes, Dev. Biol. 67 (1978) 243-248.

[19] A. Peres, G. Bernardini, The effective membrane capacity of Xenopus eggs: its relations with membrane conductance and cortical granule exocytosis, Pflugers Arch. 404 (1985) 266-272.

[20] O.P. Hamill, A. Marty, E. Neher, B. Sakmann, F.J. Sigworth, Improved patch-clamp technique for high-resolution current recording from cells and cell-free membrane patches, Pflügers Archiv Eur. J. Physiol. 391 (1981) 85-100.

[21] E. Neher, A. Marty, Discrete changes of cell membrane capacitance observed under conditions of enhanced secretion in bovine adrenal chromaffin cells, Proc. Natl. Acad. Sci. U. S. A. 79 (1982) 6712-6716.

[22] M. Lindau, E. Neher, Patch-clamp techniques for time-resolved capacitance measurements in single cells, Pflugers Arch. 411 (1988) 137-146.

[23] M. Lindau, Time-resolved capacitance measurements: monitoring exocytosis in single cells, Q. Rev. Biophys. 24 (1991) 75-101.

[24] K.D. Gillis, Techniques for membrane capacitance measurements, in: B. Sakmann, E. Neher (Eds.), Single-channel recording, Plenum Press, New York, 1995, pp. $155-198$.

[25] A. Marty, Ca-dependent K channels with large unitary conductance in chromaffin cell membranes, Nature 291 (1981) 497-500.

[26] K.D. Gillis, Admittance-based measurement of membrane capacitance using the EPC-9 patch-clamp amplifier, Pflügers Arch. 439 (2000) 655-664.

[27] D.F. Donelly, A novel method for rapid measurement of membrane resistance, capacitance, and access resistance, Biophys. J. 66 (1994) 873-877.

[28] D.W. Barnett, S. Misler, An optimized approach to membrane capacitance estimation using dual-frequency excitation, Biophys. J. 72 (1997) 1641-1658.

[29] V. Rohlicek, A. Schmid, Dual-frequency method for synchronous measurement of cell capacitance, membrane conductance and access-resistance on single cells, Pflügers Arch. 428 (1994) 30-38.

[30] R.E. Thompson, M. Lindau, W.W. Webb, Robust, high-resolution, whole cell patch-clamp capacitance measurements using square wave stimulation, Biophys. J. 81 (2001) 937-948.

[31] K.D. Gillis, R. Mößner, E. Neher, Protein kinase C enhances exocytosis from chromaffin cells by increasing the size of the readily releasable pool of secretory granules, Neuron 16 (1996) 1209-1220.

[32] F.E. Schweizer, T. Schafer, C. Tapparelli, M. Grob, U.O. Karli, R. Heumann, H. Thoenen, R.J. Bookman, M.M. Burger, Inhibition of exocytosis by intracellularly applied antibodies against a chromaffin granule-binding protein, Nature 339 (1989) 709-712.

[33] N. Fidler Lim, M.C. Nowycky, J. Bookman, Direct measurement of exocytosis and calcium currents in single vertebrate nerve terminals, Nature 344 (1990) 449-451.

[34] D.E. Clapham, E. Neher, Trifluoperazine reduces inward ionic currents and secretion by separate mechanisms in bovine chromaffin cells, J. Physiol. 353 (1984) $541-564$.

[35] L. von Rüden, E. Neher, A Ca-dependent early step in the release of catecholamines from adrenal chromaffin cells, Science 262 (1993) 1061-1065.

[36] K. Debus, J. Hartmann, G. Kilic, M. Lindau, Influence of conductance changes on patch clamp capacitance measurements using a lock-in amplifier and limitations of the phase tracking technique, Biophys. J. 69 (1995) 2808-2822.

[37] M. Lindau, E.L. Stuenkel, J.J. Nordmann, Depolarization, intracellular calcium and exocytosis in single vertebrate nerve endings, Biophys. J. 61 (1992) 19-30.

[38] G.J. Augustine, E. Neher, Calcium requirements for secretion in bovine chromaffin cells, JP 450 (1992) 247-271. 
[39] Y. Zhao, Q. Fang, S.G. Straub, M. Lindau, G.W. Sharp, Noradrenaline inhibits exocytosis via the $G$ protein betagamma subunit and refilling of the readily releasable granule pool via the alpha(i1/2) subunit, J. Physiol. 588 (2010) 3485-3498.

[40] E. Neher, G.J. Augustine, Calcium gradients and buffers in bovine chromaffin cells, J. Physiol. (1992) 1-29.

[41] F.D. Marengo, J.R. Monck, Development and dissipation of $\mathrm{Ca}(2+)$ gradients in adrenal chromaffin cells, Biophys. J. 79 (2000) 1800-1820.

[42] Z. Zhou, E. Neher, Mobile and immobile calcium buffers in bovine adrenal chromaffin cells, J. Physiol. 469 (1993) 247-273.

[43] R. Llinas, M. Sugimori, R.B. Silver, Microdomains of high calcium concentration in a presynaptic terminal, Science 256 (1992) 677-679.

[44] W.M. Roberts, R.A. Jacobs, A.J. Hudspeth, Colocalization of ion channels involved in frequency selectivity and synaptic transmission at presynaptic active zones of hair cells, J. Neurosci. 10 (1990) 3664-3684.

[45] G.J. Augustine, How does calcium trigger neurotransmitter release? Curr. Opin. Neurobiol. 11 (2001) 320-326.

[46] E. Neher, R.S. Zucker, Multiple calcium-dependent processes related to secretion in bovine chromaffin cells, Neuron 10 (1993) 21-30.

[47] P. Thomas, J.G. Wong, W. Almers, Millisecond studies of secretion in single rat pituitary cells stimulated by flash photolysis of caged $\mathrm{Ca}^{2+}$, Eur. Mol. Biol. Organiz. J. 12 (1993) 303-306.

[48] C. Heinemann, R.H. Chow, E. Neher, R.S. Zucker, Kinetics of the secretory response in bovine chromaffin cells following flash photolysis of caged $\mathrm{Ca}^{2+}$, Biochem. J. 67 (1994) 2546-2557.

[49] G.C. Ellis-Davies, J.H. Kaplan, Nitrophenyl-EGTA, a photolabile chelator that selectively binds $\mathrm{Ca} 2+$ with high affinity and releases it rapidly upon photolysis, Proc. Natl. Acad. Sci. U. S. A. 91 (1994) 187-191.

[50] G.C. Ellis-Davies, J.H. Kaplan, R.J. Barsotti, Laser photolysis of caged calcium: rates of calcium release by nitrophenyl-EGTA and DM-nitrophen, Biophys. J. 70 (1996) 1006-1016.

[51] T. Voets, Dissection of three Ca2+-dependent steps leading to secretion in chromaffin cells from mouse adrenal slices, Neuron 28 (2000) 537-545.

[52] A.M. Walter, K. Wiederhold, D. Bruns, D. Fasshauer, J.B. Sorensen, Synaptobrevin $\mathrm{N}$-terminally bound to syntaxin-SNAP-25 defines the primed vesicle state in regulated exocytosis, J. Cell Biol. 188 (2010) 401-413.

[53] T. Xu, T. Binz, H. Niemann, E. Neher, Multiple kinetic components of exocytosis distinguished by neurotoxin sensitivity, Nat. Neurosci. 1 (1998) 192-200.

[54] A.F. Oberhauser, I.M. Robinson, J.M. Fernandez, Simultaneous capacitance and amperometric measurements of exocytosis: a comparison, Biophys. J. 71 (1996) 1131-1139.

[55] T. Voets, E. Neher, T. Moser, Mechanisms underlying phasic and sustained secretion in chromaffin cells from mouse adrenal slices, Neuron 23 (1999) 607-615.

[56] L.J. Breckenridge, W. Almers, Currents through the fusion pore that forms during exocytosis of a secretory vesicle, Nature 328 (1987) 814-817.

[57] J. Zimmerberg, M. Curran, F.S. Cohen, M. Brodwick, Simultaneous electrical and optical measurements show that membrane fusion precedes secretory granule swelling during exocytosis of beige mouse mast cells, PNAS 84 (1987) 1585-1589.

[58] A.E. Spruce, L.J. Breckenridge, A.K. Lee, W. Almers, Properties of the fusion pore that forms during exocytosis of a mast cell secretory vesicle, Neuron 4 (1990) 643-654.

[59] K. Lollike, N. Borregaard, M. Lindau, The exocytotic fusion pore of small granules has a conductance similar to an ion channel, J. Cell Biol. 129 (1995) 99-104.

[60] S. Scepek, M. Lindau, Focal exocytosis by eosinophils-compound exocytosis and cumulative fusion, EMBO J. 12 (1993) 1811-1817.

[61] S. Scepek, R. Moqbel, M. Lindau, Compound exocytosis and cumulative degranulation by eosinophils and their role in parasite killing, Parasitol. Today 10 (1994) 276-278.
[62] J. Hartmann, S. Scepek, I. Hafez, M. Lindau, Differential regulation of exocytotic fusion and granule-granule fusion in eosinophils by Ca2+ and GTP analogs, J. Biol. Chem. 278 (2003) 44929-44934.

[63] I. Hafez, A. Stolpe, M. Lindau, Compound exocytosis and cumulative fusion in eosinophils, J. Biol. Chem. 278 (2003) 44921-44928.

[64] S. Scepek, J.R. Coorssen, M. Lindau, Fusion pore expansion in horse eosinophils is modulated by $\mathrm{Ca} 2+$ and protein kinase $\mathrm{C}$ via distinct mechanisms, EMBO J. 17 (1998) 4340-4345

[65] K. Debus, M. Lindau, Resolution of patch capacitance recordings and of fusion pore conductances in small vesicles, Biophys. J. 78 (2000) 2983-2997.

[66] A. Neef, C. Heinemann, T. Moser, Measurements of membrane patch capacitance using a software-based lock-in system, Pflugers Arch. 454 (2007) 335-344.

[67] L. He, L. Xue, J. Xu, B.D. McNeil, L. Bai, E. Melicoff, R. Adachi, L.G. Wu, Compound vesicle fusion increases quantal size and potentiates synaptic transmission, $\mathrm{Na}$ ture 459 (2009) 93-97.

[68] A. Albillos, G. Dernick, H. Horstmann, W. Almers, G. Alvarez de Toledo, M. Lindau, The exocytotic event in chromaffin cells revealed by patch amperometry, Nature 389 (1997) 509-512.

[69] E. Alés, L. Tabares, J.M. Poyato, V. Valero, M. Lindau, G. Alvarez de Toledo, High calcium concentrations shift the mode of exocytosis to the kiss-and-run mechanism, Nat. Cell Biol. 1 (1999) 40-44.

[70] V.A. Klyachko, M.B. Jackson, Capacitance steps and fusion pores of small and large-dense-core vesicles in nerve terminals, Nature 418 (2002) 89-92.

[71] P.E. MacDonald, S. Obermuller, J. Vikman, J. Galvanovskis, P. Rorsman, L. Eliasson, Regulated exocytosis and kiss-and-run of synaptic-like microvesicles in INS-1 and primary rat beta-cells, Diabetes 54 (2005) 736-743.

[72] A. Elhamdani, F. Azizi, C.R. Artalejo, Double patch clamp reveals that transient fusion (kiss-and-run) is a major mechanism of secretion in calf adrenal chromaffin cells: high calcium shifts the mechanism from kiss-and-run to complete fusion, J. Neurosci. 26 (2006) 3030-3036.

[73] M. Lindau, H. Rosenboom, J. Nordmann, Exocytosis and endocytosis in single peptidergic nerve terminals, Adv. Second Messenger Phosphoprotein Res. 29 (1994) 173-187.

[74] L. He, X.S. Wu, R. Mohan, L.G. Wu, Two modes of fusion pore opening revealed by cell-attached recordings at a synapse, Nature 444 (2006) 102-105.

[75] S.T. Hanna, G.M. Pigeau, J. Galvanovskis, A. Clark, P. Rorsman, P.E. MacDonald, Kiss-and-run exocytosis and fusion pores of secretory vesicles in human betacells, Pflugers Arch. 457 (2009) 1343-1350.

[76] Z. Zhang, Z. Zhang, M.B. Jackson, Synaptotagmin IV modulation of vesicle size and fusion pores in PC12 cells, Biophys. J. 98 (2010) 968-978.

[77] G. Dernick, G.A. de Toledo, M. Lindau, The patch amperometry technique: design of a method to study exocytosis of single vesicles, in: A.C. Michael, L.M. Borland (Eds.), Electrochemical Methods for Neuroscience, CRC Press, Boca Raton (FL), 2007, pp. 315-336.

[78] G. Dernick, L.W. Gong, L. Tabares, G. Alvarez de Toledo, M. Lindau, Patch amperometry: high-resolution measurements of single-vesicle fusion and release, Nat. Methods 2 (2005) 699-708.

[79] L.W. Gong, G. Alvarez De Toledo, M. Lindau, Secretory vesicles membrane area is regulated in tandem with quantal size in chromaffin cells, J. Neurosci. 23 (2003) 7917-7921.

[80] M.S. Montesinos, J.D. Machado, M. Camacho, J. Diaz, Y.G. Morales, D. Alvarez de la Rosa, E. Carmona, A. Castaneyra, O.H. Viveros, D.T. O'Connor, S.K. Mahata, R. Borges, The crucial role of chromogranins in storage and exocytosis revealed using chromaffin cells from chromogranin A null mouse, J. Neurosci. 28 (2008) 3350-3358.

[81] G. Alvarez de Toledo, R. Fernández-Chacón, J.M. Fernandez, Release of secretory products during transient vesicle fusion, Nature 363 (1993) 554-558.

[82] L.W. Gong, G.A. de Toledo, M. Lindau, Exocytotic catecholamine release is not associated with cation flux through channels in the vesicle membrane but $\mathrm{Na}^{+}$influx through the fusion pore, Nat. Cell Biol. 9 (2007) 915-922. 